\title{
Deflections and curvatures of a film-substrate structure with the presence of gradient stress in MEMS applications
}

\author{
Yin Zhang \\ State Key Laboratory of Nonlinear Mechanics (LNM), Institute of Mechanics, \\ Chinese Academy of Sciences, Beijing 100080, People's Republic of China \\ E-mail: zhangyin@lnm.imech.ac.cn
}

Received 4 September 2006, in final form 8 December 2006

Published 8 March 2007

Online at stacks.iop.org/JMM/17/753

\begin{abstract}
The close form solutions of deflections and curvatures for a film-substrate composite structure with the presence of gradient stress are derived. With the definition of more precise kinematic assumption, the effect of axial loading due to residual gradient stress is incorporated in the governing equation. The curvature of film-substrate with the presence of gradient stress is shown to be nonuniform when the axial loading is nonzero. When the axial loading is zero, the curvature expressions of some structures derived in this paper recover the previous ones which assume the uniform curvature. Because residual gradient stress results in both moment and axial loading inside the film-substrate composite structure, measuring both the deflection and curvature is proposed as a safe way to uniquely determine the residual stress state inside a film-substrate composite structure with the presence of gradient stress.
\end{abstract}

\section{Introduction}

The rigorous understanding of length-scale-dependent and process-dependent mechanical properties of structures with feature sizes ranging from $1 \mu \mathrm{m}$ to $1 \mathrm{~mm}$ is key to their successful fabrication and reliable operation [1]. Residual stress is one of those length-scale-dependent and processdependent mechanical properties and ubiquitous in microstructures, especially those fabricated using deposited films [1]. Residual stress and its gradients are frequently encountered in the film-substrate composite structure fabricated by low-pressure chemical vapor deposition (LPCVD) [2-5], etching [4, 5], sputtering [6, 7], wafer bonding [8] and epitaxial growth [9-14]. Various mechanisms and processings such as interfacial atomic diffusion [3], deposition temperature [4], doping/implantation [4, 5, 15], annealing process [4], defects like twin [7] and dislocation [8], substrate bias voltage [7], mismatch of coefficient of thermal expansion (CTE) $[6,16]$ and lattice parameters [9-14] can all contribute to the existence of residual stress and its gradients. The residual stress and its gradients can be either compressive or tensile depending on the fabrication process and technique [5]. Noyan et al demonstrated the dramatic size effect of film-substrate composite structures with a feature size of several microns due to thermal stress of CTE mismatch [6]. The existence of residual stress and its gradients changes the properties such as equilibrium (deflection and curvature) [1,9-14, 16-18], pull-in voltage and resonant frequencies, etc $[1,5]$. And those property changes are measured and utilized to find out residual stress and its gradients. Sometimes, the residual compressive stress and its gradients are so large as to reach and surpass the critical value of pitchfork bifurcation [19], the structure buckles and enters the post-buckling region $[4,20,21]$. Therefore, measuring the system buckling and monitoring its post-buckling deflection are also the effective ways of determining the residual stress and its gradients [4, 20,21].

Various methods and techniques such as interferometric profilometry [3], Raman spectroscopy [5], dynamic test (measuring the shift of resonant frequencies) [5], wafer curvature $[9,10]$, rotating beam structure sensor [15], optical lever technique [17], bulge test [22], M-test [23] and focus ion beam (FIB) Moiré technique [24] are developed for the measurement of residual stress. The advantages and disadvantages of some methods and techniques above are discussed in $[1,25]$. The model plays the role of interpreting 
the measured data and Stoney's formula [26] serves as the cornerstone of curvature-based measurement techniques. However, the applicability of Stoney's formula relies on several assumptions, which are well summarized by Freund et al [10]. Once one or some of the assumptions are violated, Stoney's formula can result in an error of 533\% [17]. Many models [9-14, 16-18, 27-30] are developed to relax one or some of the assumptions to extend Stoney's formula to a more generalized and realistic application. The following is the modified Stoney's formula on the curvature of film-substrate structure $\left(\kappa_{F}\right)$ as given by Freund et al $[9,10]$, which considers the effect of the film thickness when the substrate is relatively thin:

$$
\begin{aligned}
\kappa_{F}= & \kappa_{\mathrm{St}}\left(1+h_{f} / h_{s}\right)\left[1+4\left(h_{f} / h_{s}\right)\left(M_{f} / M_{s}\right)\right. \\
& +6\left(h_{f} / h_{s}\right)^{2}\left(M_{f} / M_{s}\right)+4\left(h_{f} / h_{s}\right)^{3}\left(M_{f} / M_{s}\right) \\
& \left.+\left(h_{f} / h_{s}\right)^{4}\left(M_{f} / M_{s}\right)^{2}\right]^{-1},
\end{aligned}
$$

where $h_{f}$ and $h_{s}$ are the film and substrate thicknesses, $M_{f}$ and $M_{s}$ are the effective Young's moduli of the film and substrate. For beam structure, $M_{f}$ and $M_{s}$ are simply the Young's moduli $E_{f}$ and $E_{s}$ of film and substrate. For plate structure bending into a cylindrical surface, $M_{f}=E_{f} /\left(1-v_{f}^{2}\right)$ and $M_{s}=E_{s} /\left(1-v_{s}^{2}\right)\left(v_{f}\right.$ and $v_{s}$ are Poisson's ratios of film and substrate) [31]. When the plate structure is in a biaxial stress state, $M_{f}=E_{f} /\left(1-v_{f}\right)$ and $M_{s}=E_{s} /\left(1-v_{s}\right)$ [9]. $\kappa_{\mathrm{St}}$ is the Stoney formula of curvature defined as follows:

$$
\kappa_{\mathrm{St}}=\frac{6 f}{M_{s} h_{s}^{2}} .
$$

$f$ is the surface stress of film (with the unit of $\mathrm{N} \mathrm{m}^{-1}$ ) when the film is extremely thin. Clearly, $\lim _{h_{f} / h_{s} \rightarrow 0} \kappa_{F}=\kappa_{\text {St }}$. Both equations (1) and (2) indicate the properties such as uniform curvature across the whole structure; only the two ratios of $h_{f} / h_{s}$ and $M_{f} / M_{s}$ affect the curvatures (together with $M_{s}$ and $h_{s}$ ); the length and boundary conditions do not have any influence on curvatures. Those modified/extended Stoney formulae also share the same properties [16, 18, 27, 30]. As analyzed by Freund et al, the assumption of uniform curvature in nonlinear large deformation range becomes extreme [10]. Recent papers by Zhang et al further prove that if the axial loading effect due to the residual stress is considered, the assumption of uniform curvature is generally invalid even in the linear small deformation range; and the curvature varies with the location, length and depends on the boundary conditions [28, 29]. However, the surface stress and residual stress due to the mismatch of lattice parameters of film and substrate in those two papers [28, 29] are constant and gradient stress case is not discussed. Usually, besides constant residual stress, gradient stresses varying through the structure thickness also exist [2, 3, 9, 15, 18, 25]. Inside a homogeneous straight beam, the tensile constant residual stress and the compressive one less than the buckling load do not generate bending moment. Under these loading scenarios, the beam can only elongate or be compressed. When the compressive constant residual stress is larger than the buckling load, a homogeneous straight beam can curl either up or down in its post-buckling state. However, as shown in figure 1 of a scanning electron microscope (SEM) photo of homogeneous microcantilevers after releasing, only curling-up is observed.

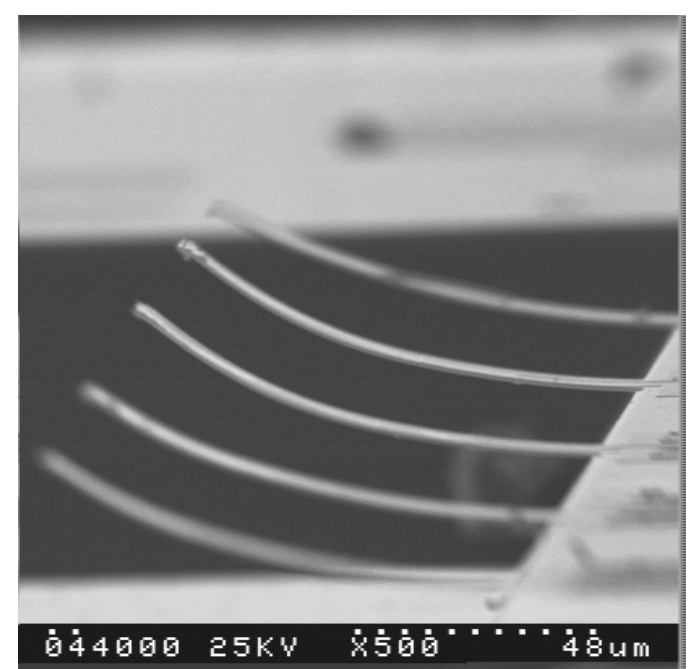

Figure 1. SEM photo of micromachined micro-beams curling up due to gradient stresses.

The stress gradients generate a bending moment, which bends the beam instantly. Residual gradient stress approximated by polynomial up to a fifth order is observed in a $2 \mu \mathrm{m}$ thick $\mathrm{p}^{+}$silicon [15]. The modified close form curvature expressions for film-substrate structure with the presence of gradient stress as derived by Freund and Suresh [9], Huang and Zhang [18] have the same properties as those discussed above for equations (1) and (2). In this paper, the assumption of uniform curvature and its error source are analyzed. The analysis of kinematic assumption shows that the assumption of uniform curvature essentially accounts for only the effect of residual stress and its gradients as a moment acting on the structure and the effect of axial loading is not incorporated. As mentioned above, the compressive axial loading due to the residual stress and its gradients are responsible for the buckling and post-buckling deflection of structure. Even when residual stress and its gradients are much smaller than the critical compressive buckling load or they are tensile, the whole system stiffness is still under the influence of the effect of axial loading due to residual stress and its gradients. In this paper, it is proved that with the presence of gradient stress inside filmsubstrate structure, the assumption of uniform curvature is not valid as far as the axial loading inside the structure is nonzero. The model development in this paper consists of two parts. One assumes the uniform curvature in the kinematic assumption and the other does not for the comparison study. The deflection and curvature due to gradient stress are both analytically derived for the linear small deformation range in the second part of model development. Some methods and techniques measure either deflection alone or curvature alone to determine the residual stress and its gradients inside the structure. As residual stress and its gradients generate both axial loading and bending moment, it is demonstrated in this paper that measuring deflection alone or curvature alone may not uniquely determine the stress state inside the structure. A systematic method of measuring both deflection and curvature to determine the residual stress state inside the structure is also presented. 


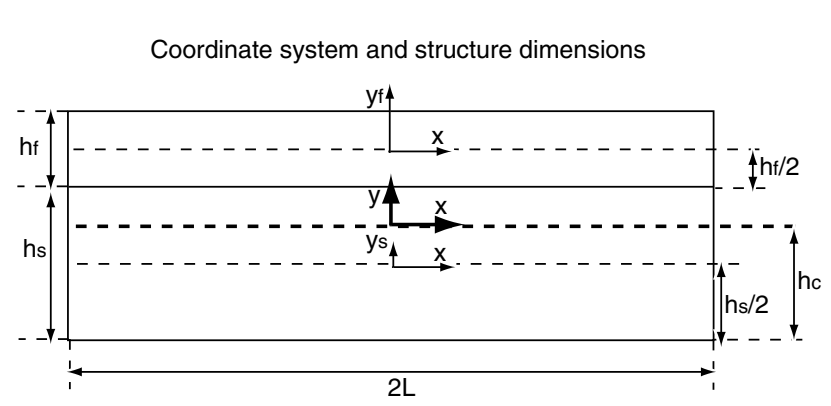

Figure 2. Schematic diagram of a film-substrate structure, its coordinate systems and related dimensions.

\section{Model development with the assumption of uniform curvature}

Figure 2 shows the coordinate system and structure dimensions. $h_{f}$ and $h_{s}$ are the film layer thickness and substrate layer thickness, respectively. The structure length is $2 L$ and $-L \leqslant x \leqslant L$. The residual gradient stress $\left(\sigma_{\text {res }}\right)$ inside the film and substrate are defined as follows [3]:

$\sigma_{\text {res }}= \begin{cases}\sigma^{f}=\sum_{k=0}^{\infty} \sigma_{k}\left(2 y_{f} / h_{f}\right)^{k}, & -h_{f} / 2 \leqslant y_{f} \leqslant h_{f} / 2 \\ \sigma^{s}=\sum_{k=0}^{\infty} \Delta_{k}\left(2 y_{s} / h_{s}\right)^{k}, & -h_{s} / 2 \leqslant y_{s} \leqslant h_{s} / 2 .\end{cases}$

The superscripts $f$ and $s$ of $\sigma$ designate film and substrate, respectively. $y_{f}$ and $y_{s}$ as shown in figure 2 start from the midplanes of the film and substrate layers, respectively. $\sigma_{0}$ and $\Delta_{0}$ are constant residual stresses inside the film and substrate. $\sigma_{k}$ and $\Delta_{k}(k \geqslant 1)$ are their gradients. Here the neutral plane of pure bending of the film-substrate bilayer is defined as follows [32]:

$$
h_{c}=\frac{M_{f} h_{f}^{2}+M_{s} h_{s}^{2}+2 M_{f} h_{f} h_{s}}{2 M_{f} h_{f}+2 M_{s} h_{s}} .
$$

The $x$-axis is the length direction and the $y$-axis is the thickness direction. The width direction is the direction perpendicular to both the $x$ and $y$ directions. $x$-coordinate here starts from the center of beam and $y$-coordinate here starts from $h_{c}$. For the case of Freund et al [9], the $y$-coordinate starts from the mid-plane of the substrate layer; Timoshenko's starts from the film-substrate interface [33] and both yield the same curvature expression [29]. The $y$-coordinate of Vanamu et al [11] starts from the neutral plane and it is shown later in this section that their curvature expression is also the same as that obtained by Freund et al and Timoshenko. The neutral plane position $h_{c}$ is irrelevant to the final result of curvature if axial loading is not considered [27]; in other words, it does not matter where you start the $y$-coordinate when there is no axial force. However, later for us to consider the axial loading, defining the neutral plane is vital to express bending strain correctly. Now in the $x-y$ coordinate system, the residual gradient stress is rewritten as follows:

$$
\sigma_{\mathrm{res}}=\left\{\begin{array}{c}
\sigma^{f}=\sum_{k=0}^{\infty} \sigma_{k}\left[y-\left(h_{f} / 2+h_{s}-h_{c}\right)\right]^{k}\left(2 / h_{f}\right)^{k} \\
h_{s}-h_{c} \leqslant y \leqslant h_{f}+h_{s}-h_{c} \\
\sigma^{s}=\sum_{k=0}^{\infty} \Delta_{k}\left[y-\left(h_{s} / 2-h_{c}\right)\right]^{k}\left(2 / h_{s}\right)^{k} \\
-h_{c} \leqslant y \leqslant h_{s}-h_{c} .
\end{array}\right.
$$

Therefore, the residual strain $\left(\epsilon_{\text {res }}\right)$ is as follows:

$\epsilon_{\mathrm{res}}= \begin{cases}\epsilon_{f}=\sigma^{f} / M_{f} & h_{s}-h_{c} \leqslant y \leqslant h_{f}+h_{s}-h_{c} \\ \epsilon_{s}=\sigma^{s} / M_{s} & -h_{c} \leqslant y \leqslant h_{s}-h_{c} .\end{cases}$

With the assumption of uniform curvature, the following kinematic assumption applies [9, 18]:

$\epsilon= \begin{cases}\epsilon_{o}+\epsilon_{f}-\kappa y & h_{s}-h_{c} \leqslant y \leqslant h_{f}+h_{s}-h_{c} \\ \epsilon_{o}+\epsilon_{s}-\kappa y & -h_{c} \leqslant y \leqslant h_{s}-h_{c} .\end{cases}$

Here $\kappa$ is the curvature and $-\kappa y$ is the bending strain. As the coordinate systems are defined differently, $\epsilon_{o}$ has different physical meanings [9, 18]. Its physical meaning in this coordinate system will be discussed later. Right now, $\epsilon_{o}$ and $\kappa$ are two independent constants to be determined. The total potential energy of the system is given as follows:

$$
\begin{aligned}
U= & \frac{1}{2} \int_{-L}^{L}\left[\int_{h_{s}-h_{c}}^{h_{s}+h_{f}-h_{c}} M_{f}\left(\epsilon_{o}+\epsilon_{f}-\kappa y\right)^{2} \mathrm{~d} y\right. \\
& \left.+\int_{-L}^{L} \int_{-h_{c}}^{h_{s}-h_{c}} M_{s}\left(\epsilon_{o}+\epsilon_{s}-\kappa y\right)^{2} \mathrm{~d} y\right] \mathrm{d} x \\
= & {\left[K_{1} \kappa+2 K_{2} \epsilon_{o}+4 K_{3} \epsilon_{o} \kappa+K_{4} \kappa^{2}+4 K_{5} \epsilon_{o}^{2}\right] L . }
\end{aligned}
$$

$K_{i} \mathrm{~s}(i=1$ to 5$)$ are defined as

$$
\begin{aligned}
K_{1}= & -\frac{h_{s}^{2}}{2} \sum_{k=0}^{\infty} \frac{\Delta_{k}}{k+2}\left[1^{k}-(-1)^{k}\right]-h_{s}\left(\frac{h_{s}}{2}-h_{c}\right) \\
& \times \sum_{k=0}^{\infty} \frac{\Delta_{k}}{k+1}\left[1^{k}+(-1)^{k}\right]-\frac{h_{s}^{2}}{2} \sum_{k=0}^{\infty} \frac{\sigma_{k}}{k+2}\left[1^{k}-(-1)^{k}\right] \\
& -h_{f}\left(\frac{h_{f}}{2}+h_{s}-h_{c}\right) \sum_{k=0}^{\infty} \frac{\sigma_{k}}{k+1}\left[1^{k}+(-1)^{k}\right],
\end{aligned}
$$

$K_{2}=\frac{h_{f}}{2} \sum_{k=0}^{\infty} \frac{\sigma_{k}}{k+1}\left[1^{k}+(-1)^{k}\right]+\frac{h_{s}}{2} \sum_{k=0}^{\infty} \frac{\Delta_{k}}{k+1}\left[1^{k}+(-1)^{k}\right]$,

$K_{3}=-\frac{M_{f}\left(h_{f}^{2}+2 h_{f} h_{s}-2 h_{f} h_{c}\right)}{2}-\frac{M_{s}\left(h_{s}^{2}-2 h_{s} h_{c}\right)}{2}$

$K_{4}=\frac{M_{f}}{3}\left[\left(h_{f}+h_{s}-h_{c}\right)^{3}-\left(h_{s}-h_{c}\right)^{3}\right]$

$+\frac{M_{s}}{3}\left[\left(h_{s}-h_{c}\right)^{3}+h_{c}^{3}\right]$,

$K_{5}=\frac{M_{f} h_{f}+M_{s} h_{s}}{4}$.

Physically, $-K_{1} / 2$ and $K_{2}$ are the moment per unit width and the axial force per unit width acting on the film-substrate bilayer due to the residual gradient stress, respectively. With the substitution of $h_{c}$ defined in equation (4), it is not that difficult to show that $K_{3}=0 . \quad K_{4}$ is the bilayer bending stiffness per unit width, $4 K_{5}$ is the axial stiffness of the bilayer per unit width. $K_{4}$ and $K_{5}$ are independent of residual stresses. 
The equilibrium requires the total energy stationary with respect to $\epsilon_{o}$ and $\kappa[9,18]$, i.e.,

$$
\partial U / \partial \epsilon_{o}=0, \quad \partial U / \partial \kappa=0,
$$

which results in the following two equations:

$$
\left\{\begin{array}{l}
4 K_{5} \epsilon_{o}+2 K_{3} \kappa=-K_{2} \\
4 K_{3} \epsilon_{o}+2 K_{4} \kappa=-K_{1} .
\end{array}\right.
$$

$\epsilon_{o}$ and $\kappa$ are thus solved as

$$
\begin{gathered}
\epsilon_{o}=\frac{-K_{2} K_{4}+K_{1} K_{3}}{4 K_{4} K_{5}-8 K_{3}^{2}}=-\frac{K_{2}}{4 K_{5}}, \\
\kappa=\frac{-K_{1} K_{5}+K_{2} K_{3}}{2 K_{4} K_{5}-K_{3}^{2}}=-\frac{K_{1}}{2 K_{4}} .
\end{gathered}
$$

In the above solutions, $K_{3}=0$ is used. $\epsilon_{o}$ is the negative axial strain when an axial loading of $K_{2}$ is applied to the film-substrate bilayer. $\kappa$ is a constant value (uniform) across the whole structure span and, clearly from equation (12), $\kappa$ depends only on the bending moment $\left(K_{1}\right)$ and bending stiffness $K_{4}$. The axial loading $K_{2}$ is only associated with $\epsilon_{o}$, the structure length does not have any influence on $\epsilon_{o}$ and $\kappa$ though it appears in the total potential energy expression of equation (8). In the derivation above, boundary conditions are neither derived nor applied. It is concluded that the curvature solution of equation (12) is independent of axial loading, length and boundary conditions. The problem results from equations (7) and (10), which assume the uniform curvature and then apply it. This problem will be fixed in the next section. Equations (7) and (10) play a central role in the curvature derivation of Freund and Suresh [9] and Huang and Zhang [18]. Let us first look at how the curvature $\kappa$ in equation (12) is related with the previous one of equation (1).

The case of epitaxial growth of film on substrate, for which equation (1) is originally derived [9], is selected to have a comparison study for the convenience of statement. Because the stress free lattice dimensions of the film and substrate are different and epitaxial growth requires the continuation of the registry or alignment of crystallographic atom positions in the single crystal substrate into the single crystal film [9], the film-substrate bilayer will deflect because of the stress/strain due to the lattice mismatch. The strain of lattice mismatch is defined as follows [9]:

$$
\epsilon_{m}=\frac{a_{s}-a_{f}}{a_{f}}
$$

Here $a_{s}$ and $a_{f}$ are the lattice dimensions parallel to the interface. For this case, the stresses/strains inside both film and substrate are constant in average sense. The strains $\epsilon_{f}$ and $\epsilon_{s}$ of the film and substrate due to lattice mismatch can be determined from the following two equations [9]:

$$
M_{f} \epsilon_{f} h_{f}+M_{s} \epsilon_{s} h_{s}=0, \quad \epsilon_{f}-\epsilon_{s}=\epsilon_{m} .
$$

The first equation is Newton's third law and the second one is the compatibility condition to guarantee the epitaxial growth. Equation (14) determines the strain distribution inside the film and substrate, which is often referred to as strain partitioning [12-14]. The strain partitioning in compliant structures can reduce the strain energy inside; therefore, the critical thickness at which dislocation is formed can be significantly enlarged [12-14]. Clearly, $\epsilon_{f}$ and $\epsilon_{s}$ are solved as constants from equation (14). Correspondingly, for the gradient stress defined in equation (5), only constant terms are kept; therefore $\sigma^{f}=\sigma_{0}=M_{f} \epsilon_{f}$ and $\sigma^{s}=\Delta_{0}=M_{s} \epsilon_{s}$. From equation (14), $\sigma_{0}$ and $\Delta_{0}$ have the following expressions:

$$
\sigma_{0}=\frac{h_{s} M_{f} M_{s} \epsilon_{m}}{M_{f} h_{f}+M_{s} h_{s}}, \quad \Delta_{0}=-\frac{h_{f}}{h_{s}} \sigma_{0} .
$$

$K_{1}$ and $K_{2}$ defined in equation (9) are now

$$
\begin{aligned}
K_{1} & =-h_{s}\left(h_{s}-2 h_{c}\right) \Delta_{0}-h_{f}\left(h_{f}+2 h_{s}-2 h_{c}\right) \sigma_{0} \\
& =-\sigma_{0} h_{f}\left(h_{f}+h_{s}\right), \\
K_{2} & =\Delta_{0} h_{s}+\sigma_{0} h_{f}=M_{f} \epsilon_{f} h_{f}+M_{s} \epsilon_{s} h_{s}=0 .
\end{aligned}
$$

With the substitution of $h_{c}$ of equation (4) into equation (12), the curvature $\kappa$ now has the following expression:

$$
\begin{aligned}
& \kappa=-\frac{K_{1}}{2 K_{4}}= \\
& \frac{6 \sigma_{0} h_{f}\left(M_{f} h_{f}+M_{s} h_{s}\right)\left(h_{f}+h_{s}\right)}{M_{s}^{2} h_{s}^{4}+4 M_{s} M_{f} h_{f} h_{s}^{3}+6 M_{s} M_{f} h_{f}^{2} h_{s}^{2}+4 M_{s} M_{f} h_{f}^{3} h_{s}+M_{f}^{2} h_{f}^{4}} .
\end{aligned}
$$

Substituting $\sigma_{0}$ of equation (15) into equation (17), it is found that

$$
\begin{aligned}
\kappa= & -\frac{K_{1}}{2 K_{4}}=\kappa_{F}=\kappa_{\mathrm{St}}\left(1+h_{f} / h_{s}\right) \\
& \times\left[1+4\left(h_{f} / h_{s}\right)\left(M_{f} / M_{s}\right)+6\left(h_{f} / h_{s}\right)^{2}\left(M_{f} / M_{s}\right)\right. \\
& \left.+4\left(h_{f} / h_{s}\right)^{3}\left(M_{f} / M_{s}\right)+\left(h_{f} / h_{s}\right)^{4}\left(M_{f} / M_{s}\right)^{2}\right]^{-1}
\end{aligned}
$$

with the definition of $\kappa_{\mathrm{St}}=6 f /\left(M_{s} h_{s}^{2}\right)=6 M_{f} h_{f} \epsilon_{m} /$ $\left(M_{s} h_{s}^{2}\right)$. So the curvature derived from equation (12) recovers the curvature expression derived by Freund et al [9, 10] when the residual stress inside both film and substrate is constant and the constraint conditions of equation (14) hold. Equation (14) prescribes that the axial loading of the whole film-substrate bilayer due to epitaxial growth is zero. Generally, the residual stress and its gradients inside the film and those inside the substrate are independent of each other. As the result, the axial loading inside film-substrate bilayer may not be zero. The curvature expression in equation (12) is a more general solution, which includes the effects of both thickness and gradient stress though the effect of gradient stress is only embodied in the bending moment $K_{1}$. The curvature expression obtained by Vanamu et al [11] for the constant residual stress and zero axial loading case is as follows:

$$
\kappa=\frac{M_{\text {bending }}^{s}-M_{\text {bending }}^{f}}{M_{s} I_{s}+M_{f} I_{f}} .
$$

The coordinate system of Vanamu et al [11] is the same as the one used in this paper, i.e., $y$ starts from the neutral axis. $M_{\text {bending }}^{s}$ and $M_{\text {bending }}^{f}$ are the bending moments generated by the substrate and film, respectively. Therefore, $M_{\text {bending }}^{s}-$ $M_{\text {bending }}^{f}=-K_{1} / 2 . I_{s}$ and $I_{f}$ are the moments of inertia per unit width of the substrate and film, respectively. Physically $M_{s} I_{s}+M_{f} I_{f}$ is the effective bending stiffness of the filmsubstrate bilayer, which is $K_{4}$. So we can conclude here that $\kappa=\left(M_{\text {bending }}^{s}-M_{\text {bending }}^{f}\right) /\left(M_{s} I_{s}+M_{f} I_{f}\right)=-K_{1} /\left(2 K_{4}\right)$. 
Deflections and curvatures of a film-substrate structure with the presence of gradient stress

\section{Model development without the assumption of uniform curvature}

The curvature of film-substrate structure is related to its deflection $v$ as follows:

$$
\kappa=\frac{1}{R}=v_{x x} /\left(1+v_{x}^{2}\right)^{\frac{3}{2}}
$$

Here $R$ is the radius of curvature and $v_{x x}=\mathrm{d}^{2} v / \mathrm{d} x^{2}$. For small deflection, $\kappa \approx v_{x x}$. The assumption of uniform curvature in other words is to require that the deflection of the filmsubstrate bilayer is an arc of a circle (or parabola if curvature is approximated as $v_{x x}$ ). There is no mechanism to guarantee such deflection for different residual gradient stresses. In this section, the structure deflection $v$ is used as an unknown variable and curvature is defined as $\kappa=v_{x x}$. Without the assumption of uniform curvature, the following new kinematic assumption applies:

$\epsilon= \begin{cases}\epsilon_{f}-y v_{x x}+v_{x}^{2} / 2 & h_{s}-h_{c} \leqslant y \leqslant h_{f}+h_{s}-h_{c} \\ \epsilon_{s}-y v_{x x}+v_{x}^{2} / 2 & -h_{c} \leqslant y_{s} \leqslant h_{s}-h_{c} .\end{cases}$

The biggest difference between equations (6) and (21) is that there is no such assumption of uniform curvature. The curvature of $v_{x x}$ may vary as $x$ changes. $v_{x}^{2} / 2$ is the counterpart of $\epsilon_{o}$. Instead of a constant value as $\epsilon_{o}, v_{x}^{2} / 2$ may also vary as $x$ changes. Although $v_{x}^{2} / 2$ is a nonlinear term in equation (21), it will result in a linear term in the governing equation, as shown later. In fact, the above kinematic assumption is used by Freund et al for the large nonlinear deflection range [10]. It will be shown that, even in the linear small deflection range, the kinematic assumption of equation (21) must be used; otherwise serious errors can be generated. Actually, equation (21) is only suitable for the linear small deflection case and equation (21) cannot be used for the study of large deflection in the post-buckling region $[20,21]$. The more complex and precise kinematic assumption for large deflection and related discussion can be found in Thurman and Mote's paper [34]. In equation (21), only one deflection variable $v$ is used, which implicitly assume that the film and substrate share a common curvature $v_{x x}$. This one deflection variable also guarantees the continuity of the displacements of film and substrate at the interface, i.e., there is no interfacial slip or the interface is perfect. Compared with the perfect interface case, the interfacial slip can result in a very different stress distribution inside the film and substrate [6]; therefore the deflection and curvature of the film-substrate bilayer with interfacial slip can be very different. Now the total potential energy is

$$
\begin{aligned}
U= & \frac{1}{2} \int_{-L}^{L}\left[\int_{h_{s}-h_{c}}^{h_{s}+h_{f}-h_{c}} M_{f}\left(\epsilon_{f}-y v_{x x}+v_{x}^{2} / 2\right)^{2} \mathrm{~d} y\right. \\
& \left.+\int_{-L}^{L} \int_{-h_{c}}^{h_{s}-h_{c}} M_{s}\left(\epsilon_{s}-y v_{x x}+v_{x}^{2} / 2\right)^{2} \mathrm{~d} y\right] \mathrm{d} x .
\end{aligned}
$$

With the application of the principle of minimum potential energy (PMPE), which is $\delta U=0$, the governing equation of film-substrate structure deflection is derived as

$$
K_{4} v_{x x x x}-K_{2} v_{x x}=0 .
$$

$K_{3}=0$ is used during derivation. For the cantilever beam, the boundary conditions are derived as

$\left\{\begin{array}{l}v=0, \quad v_{x}=0, \quad x=-L \\ K_{2} v_{x}-K_{4} v_{x x x}=0, \quad K_{1}+2 K_{4} v_{x x}=0, \quad x=L .\end{array}\right.$

For the hinged-hinged beam, the boundary conditions are

$$
\left\{\begin{array}{lll}
v=0, & K_{1}+2 K_{4} v_{x x}=0, & x=-L \\
v=0, & K_{1}+2 K_{4} v_{x x}=0, & x=L
\end{array}\right.
$$

For the free-free beam, the boundary conditions are derived as

$$
\begin{cases}K_{1}+2 K_{4} v_{x x}=0, & x=-L, L \\ K_{2} v_{x}-K_{4} v_{x x x}=0, & x=-L, L .\end{cases}
$$

For the clamped-hinged beam, the boundary conditions are derived as

$$
\left\{\begin{array}{lll}
v=0, & v_{x}=0, & x=-L, \\
v=0, & K_{1}+2 K_{4} v_{x x}=0, & x=L .
\end{array}\right.
$$

With the definition of $\beta=\sqrt{\left|\frac{K_{2}}{K_{4}}\right|}$, equation (23) has the following solution form for the different $K_{2}$ values of axial loading:

$v(x)= \begin{cases}C_{1} \cosh (\beta x)+C_{2} \sinh (\beta x)+C_{3} x+C_{4}, & K_{2}>0 \\ C_{1} \cos (\beta x)+C_{2} \sin (\beta x)+C_{3} x+C_{4}, & K_{2}<0 \\ C_{1} x^{3}+C_{2} x^{2}+C_{3} x+C_{4}, & K_{2}=0 .\end{cases}$

$C_{i}$ s $(i=1$ to 4$)$ are the constants to be determined by boundary conditions. With the use of boundary conditions, the deflection solution of the cantilever beam is

$v(x)= \begin{cases}\frac{-K_{1}}{2 K_{4} \cosh (2 \beta L) \beta^{2}}[\cosh (\beta x+\beta L)-1], & K_{2}>0 \\ \frac{K_{1}}{2 K_{4} \cos (2 \beta L) \beta^{2}}[\cos (\beta x+\beta L)-1], & K_{2}<0 \\ \frac{-K_{1}}{4 K_{4}}\left(x^{2}+2 L x+L^{2}\right), & K_{2}=0 .\end{cases}$

And the curvature of the cantilever beam is

$$
\kappa=v_{x x}= \begin{cases}\frac{-K_{1}}{2 K_{4} \cosh (2 \beta L)} \cosh (\beta x+\beta L), & K_{2}>0 \\ \frac{-K_{1}}{2 K_{4} \cos (2 \beta L)} \cos (\beta x+\beta L), & K_{2}<0 \\ \frac{-K_{1}}{2 K_{4}}, & K_{2}=0 .\end{cases}
$$

The deflection solution of the hinged-hinged beam is

$$
v(x)= \begin{cases}\frac{-K_{1}}{2 K_{4} \cosh (\beta L) \beta^{2}}[\cosh (\beta x)-\cosh (\beta L)], & K_{2}>0 \\ \frac{K_{1}}{2 K_{4} \cos (\beta L) \beta^{2}}[\cos (\beta x)-\cos (\beta L)], & K_{2}<0 \\ \frac{-K_{1}}{4 K_{4}}\left(x^{2}-L^{2}\right), & K_{2}=0 .\end{cases}
$$

And the curvature of the hinged-hinged beam is

$$
\kappa=v_{x x}= \begin{cases}\frac{-K_{1}}{2 K_{4} \cosh (\beta L)} \cosh (\beta x), & K_{2}>0 \\ \frac{-K_{1}}{2 K_{4} \cos (\beta L)} \cos (\beta x), & K_{2}<0 \\ \frac{-K_{1}}{2 K_{4}}, & K_{2}=0 .\end{cases}
$$


For the free-free beam, the deflection is

$$
v(x)= \begin{cases}\frac{-K_{1}}{2 K_{4} \cosh (\beta L) \beta^{2}} \cosh (\beta x)+C_{3} x+C_{4}, & K_{2}>0 \\ \frac{K_{1}}{2 K_{4} \cos (\beta L) \beta^{2}} \cos (\beta x)+C_{3} x+C_{4}, & K_{2}<0 \\ \frac{-K_{1}}{4 K_{4}} x^{2}+C_{3} x+C_{4}, & K_{2}=0 .\end{cases}
$$

For the free-free beam, $C_{3}$ and $C_{4}$ cannot be determined via the boundary conditions alone. $C_{3} x+C_{4}$ physically stands for the rigid body displacement, which has no contribution to the total potential energy. The curvature of the free-free beam is the same as that of the hinged-hinged beam in equation (32). The deflection solution of the clamped-hinged beam is

$$
\begin{aligned}
& v(x)= \\
& \left\{\begin{array}{c}
\left\{-K_{1}\{\beta \sinh (\beta L)[\sinh (\beta x)-\sinh (\beta L) x / L]\right. \\
\quad+[\beta \cosh (\beta L)-\sinh (\beta L) / L][\cosh (\beta x)-\cosh (\beta L)]\}\} / \\
\left\{2 K_{4}\left[\beta^{3} \cosh (2 \beta L)-\beta^{2} \sinh (2 \beta L) /(2 L)\right]\right\}, \quad K_{2}>0 \\
\left\{-K_{1}\{\beta \sin (\beta L)[\sin (\beta x)-\sin (\beta L) x / L]\right. \\
\quad-[\beta \cos (\beta L)-\sin (\beta L) / L][\cos (\beta x)-\cos (\beta L)]\}\} / \\
\left\{2 K_{4}\left[\beta^{3} \cos (2 \beta L)-\beta^{2} \sin (2 \beta L) /(2 L)\right]\right\}, \quad K_{2}<0 \\
-K_{1}\left[\frac{1}{16 K_{4}}\left(x^{3}-L^{2} x\right)+x^{2}-L^{2}\right], \quad K_{2}=0 .
\end{array}\right.
\end{aligned}
$$

And the clamped-hinged beam curvature is

$$
\begin{aligned}
& \kappa=v_{x x}= \\
& \left\{\begin{array}{l}
\left\{-K_{1}\left\{\beta^{3} \sinh (\beta L) \sinh (\beta x)+[\beta \cosh (\beta L)\right.\right. \\
\left.\left.\quad-\sinh (\beta L) / L] \beta^{2} \cosh (\beta x)\right\}\right\} / \\
\left\{2 K_{4}\left[\beta^{3} \cosh (2 \beta L)-\beta^{2} \sinh (2 \beta L) /(2 L)\right]\right\}, \quad K_{2}>0 \\
\left\{-K_{1}\left\{-\beta^{3} \sin (\beta L) \sin (\beta x)+[\beta \cos (\beta L)\right.\right. \\
\left.\left.\quad-\sin (\beta L) / L] \beta^{2} \cos (\beta x)\right\}\right\} / \\
\left\{2 K_{4}\left[\beta^{3} \cos (2 \beta L)-\beta^{2} \sin (2 \beta L) /(2 L)\right]\right\}, \quad K_{2}<0 \\
\frac{-K_{1}}{2 K_{4}}\left(\frac{3 x}{4 L}+\frac{1}{4}\right), \quad K_{2}=0 .
\end{array}\right.
\end{aligned}
$$

Clearly, for all the structures here when $K_{2} \neq 0$, the curvature $\kappa$ is a function of $x$ and its expression differs for different boundary conditions. The axial loading $K_{2}(\beta)$ determines the solution form of both the deflection and curvature when $K_{2} \neq 0$. $L$ also explicitly appears in the expressions of both deflection and curvature. Only when there is no axial loading $\left(K_{2}=0\right)$, the curvature of cantilever, hinged-hinged and free-free beams is the constant value of $-K_{1} / 2 K_{4}$, which recovers the curvature expression obtained by the model with the assumption of uniform curvature [18]. The exceptional case is the clamped-hinged beam; its curvature is not a constant when $K_{2}=0$.

\section{Results and discussions}

In this paper, $M_{f}=142 \mathrm{GPa}$ for germanium and $M_{s}=$ $180.5 \mathrm{GPa}$ for silicon [9]; $h_{f}$ and $h_{s}$ are fixed as $h_{f}=1 \mu \mathrm{m}$ and $h_{s}=2 \mu \mathrm{m}$. Once Young's moduli and thickness of the

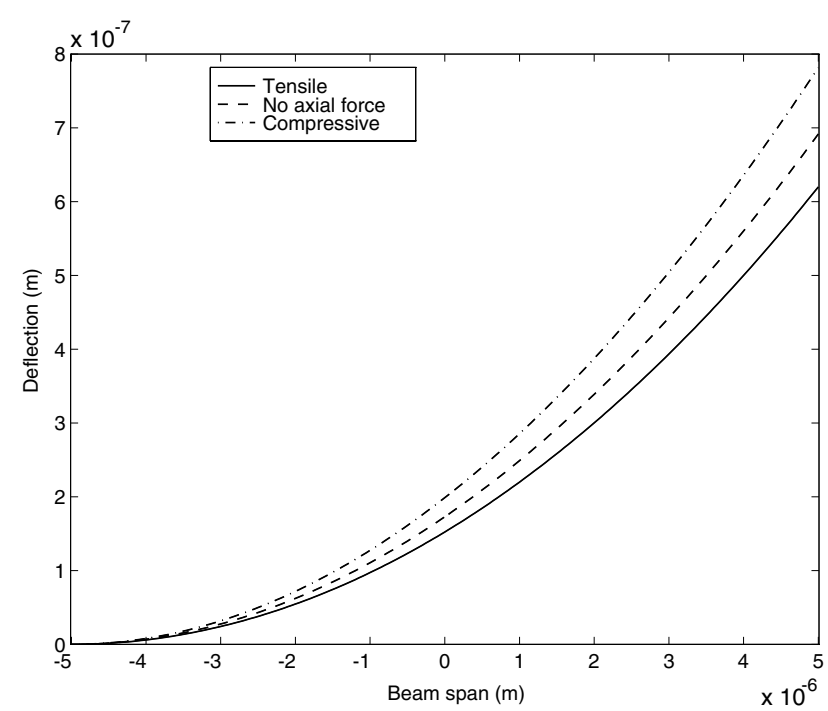

Figure 3. The three deflections along the beam span of a cantilever beam when the beam is under tensile, no axial load and compressive loadings, respectively.

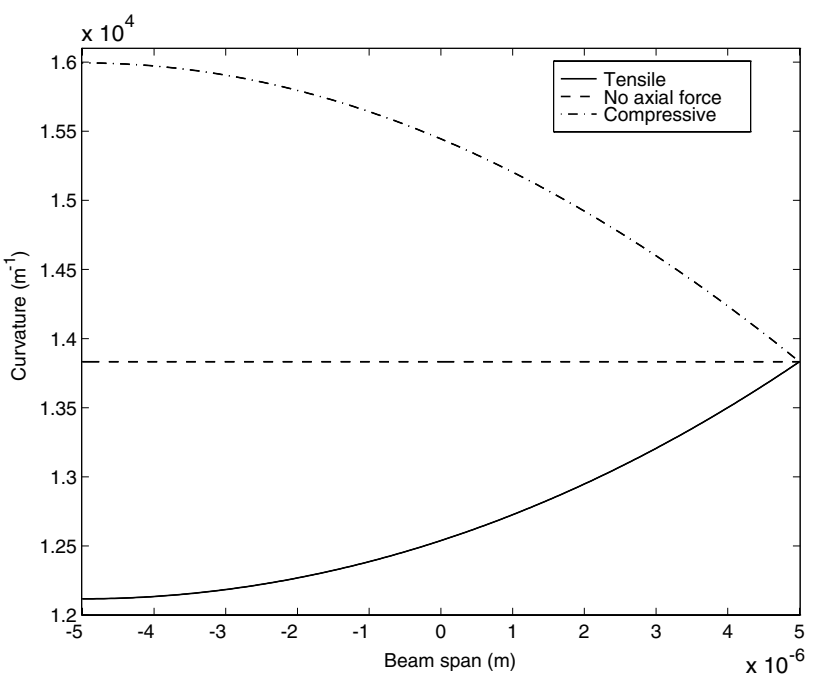

Figure 4. The three curvatures along the beam span of a cantilever beam when the beam is under tensile, no axial load and compressive loadings, respectively.

film and substrate are given, $K_{4}$ and $K_{5}$ can be calculated from equation (9). From figures 3 to figure 8 , the structure length is fixed as $2 L=10 \mu \mathrm{m}, K_{1}=-1 \times 10^{-2} \mathrm{~N}$ (moment per unit width), the axial loading $K_{2}$ varies as $K_{2}=1 \times$ $10^{3} \mathrm{~N} \mathrm{~m}^{-1}$ (force per unit width) for the tensile axial loading case, $K_{2}=-1 \times 10^{3} \mathrm{~N} \mathrm{~m}^{-1}$ for the compressive axial loading case and $K_{2}=0 \mathrm{~N} \mathrm{~m}^{-1}$ for the no axial loading case.

Figures 3 and 4 show the deflections and curvatures of the cantilever beam under tensile, compressive and no axial loadings. The deflection and curvature under tensile loading are always the smallest and those under compressive loading are always the largest because tension stiffens the structure and compression softens the structure. Only at the free end at $x=L$, the three cases have the same curvature value of $-K_{1} / 2 K_{4}$. Figures 5 and 6 show the deflections and curvatures of the hinged-hinged beam under tensile, 


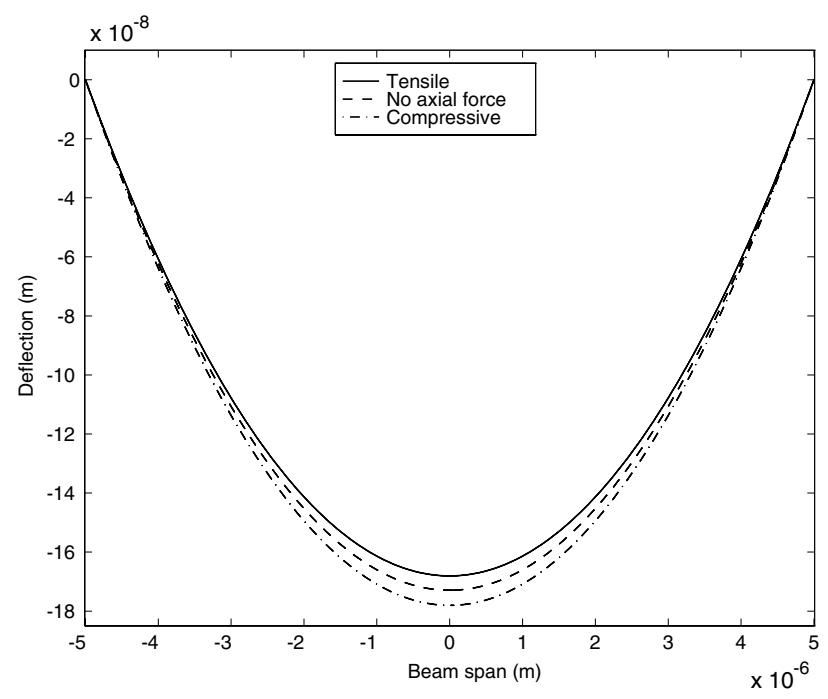

Figure 5. The three deflections along the beam span of a hinged-hinged beam when the beam is under tensile, no axial load and compressive loadings, respectively.

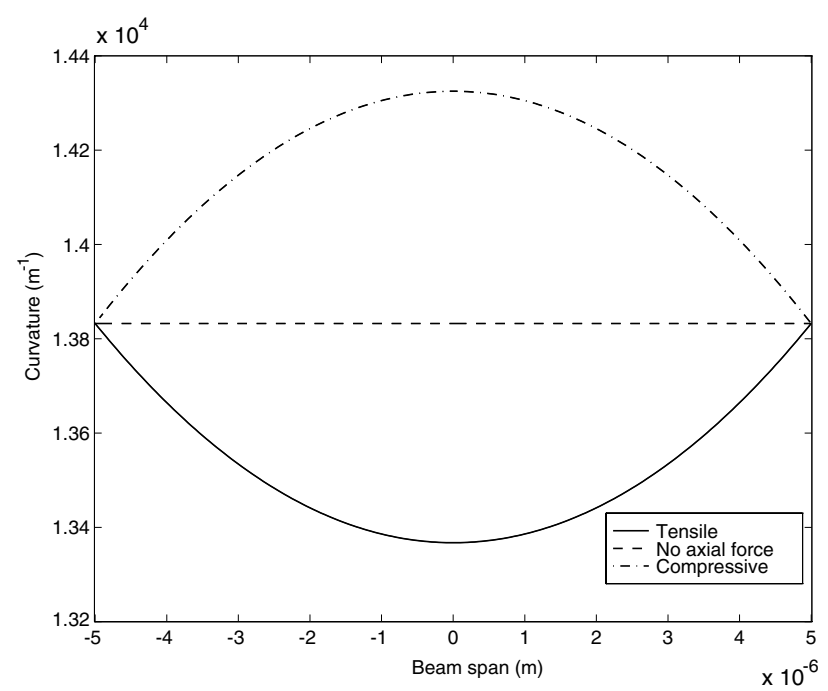

Figure 6. The three curvatures along the beam span of a hinged-hinged beam when the beam is under tensile, no axial load and compressive loadings, respectively.

compressive and no axial loadings. At the two hinged ends, the curvatures of the three loading cases have the same value of $-K_{1} / 2 K_{4}$. Figures 7 and 8 show the deflections and curvatures of the clamped-hinged beam under tensile, compressive and no axial loadings. Although the curvature expressions of equation (35) are nonlinear when $K_{2} \neq 0$, they look linear in figure 8 . All three curvatures in figure 8 monotonously increase and at the hinged end of $x=L$ they reach the maximum value of $-K_{1} / 2 K_{4}$. Figure 3-8 demonstrate the effect of boundary conditions on deflection and curvature for the structure under the same loading scenarios. Except the clamped-hinged beam, the curvature is uniform when $K_{2}=0$; boundary conditions and length have no influence. Figures 9 and 10 demonstrate the length effect on deflection and curvature. The two hingedhinged beams with different lengths of $2 L=10 \mu \mathrm{m}$ and $2 L=14 \mu \mathrm{m}$ are under the same loadings of $K_{1}$ and $K_{2}$.

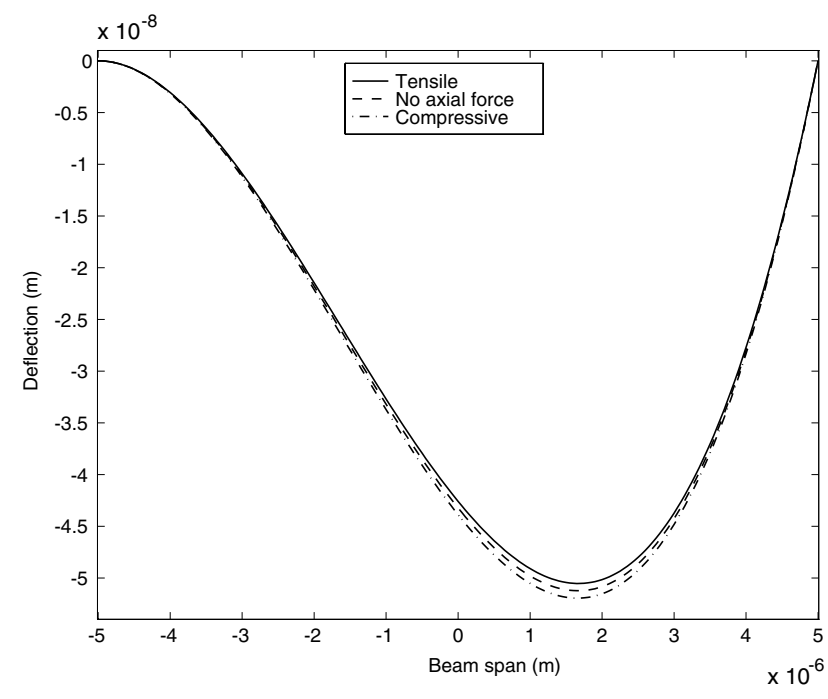

Figure 7. The three deflections along the beam span of a clamped-hinged beam when the beam is under tensile, no axial load and compressive loadings, respectively.

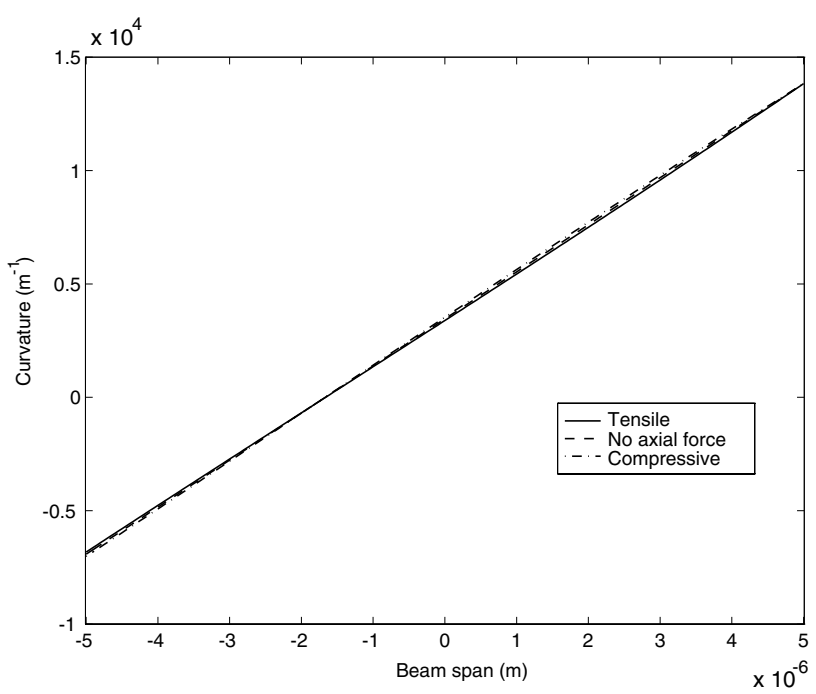

Figure 8. The three curvatures along the beam span of a clamped-hinged beam when the beam is under tensile, no axial load and compressive loadings, respectively.

Clearly, the deflection and curvature of the beam with larger length are more sensitive to the loadings and vary with larger amplitude.

As residual gradient stresses generate both axial loading and bending moment, different combinations of axial loading and bending moment can result in the almost same deflection curve as shown in figure 11. Figure 11 plots two deflection curves of a hinged-hinged beam under two different loading sets of $K_{1}=-1 \times 10^{-2} \mathrm{~N}, K_{2}=1 \times 10^{3} \mathrm{~N} \mathrm{~m}^{-1}$ and $K_{1}=$ $-1.085 \times 10^{-2} \mathrm{~N}, K_{2}=4 \times 10^{3} \mathrm{~N} \mathrm{~m}^{-1}$, respectively. There is almost no difference between the two deflection curves and measuring the deflection alone [16] is thus not sufficient to determine the residual stress state. Although the deflection curves are very similar, the curvature curves look dramatically different for the two loading cases as shown in figure 12 . With the existence of gradient stresses generating the axial 


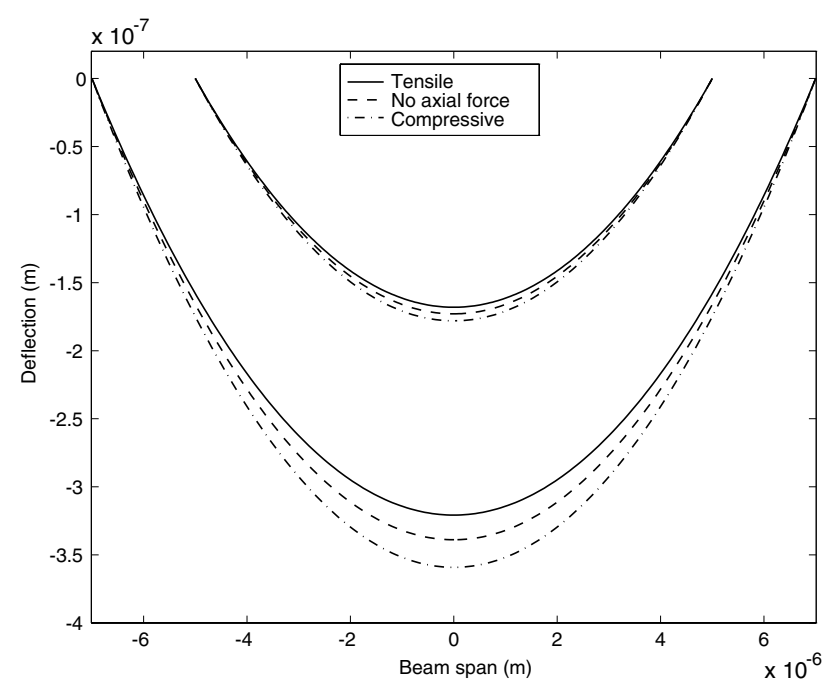

Figure 9. The deflection comparison of two hinged-hinged beams under the same loadings of $K_{1}=-1 \times 10^{-2} \mathrm{~N}$ and $K_{2}=1 \times$ $10^{3} \mathrm{~N} \mathrm{~m}^{-1}$. The lengths of the two beams are $2 L=10 \mu \mathrm{m}$ and $2 L=14 \mu \mathrm{m}$, respectively.

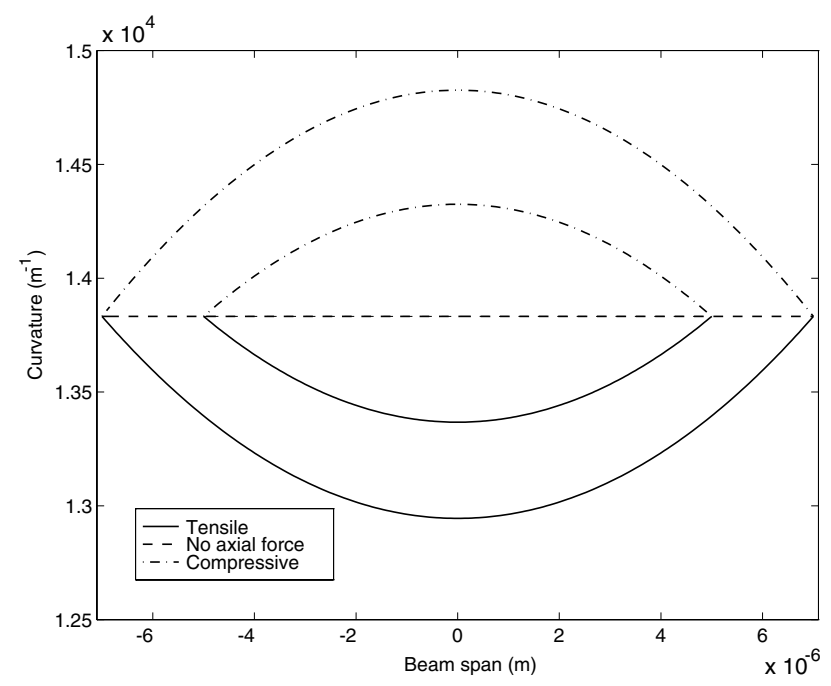

Figure 10. The curvature comparison of two hinged-hinged beams under the same loadings of $K_{1}=-1 \times 10^{-2} \mathrm{~N}$ and $K_{2}=1 \times$ $10^{3} \mathrm{~N} \mathrm{~m}^{-1}$. The lengths of the two beams are $2 L=10 \mu \mathrm{m}$ and $2 L=14 \mu \mathrm{m}$, respectively.

loading and bending moment, it is suggested to measure both deflection and curvature to determine the stress state. Figures 13 and 14 show the deflection and curvature at $x=0$ of a hinged-hinged beam as functions of $K_{1}$ and $K_{2}$. The structure length is also fixed as $2 L=10 \mu \mathrm{m} .-\pi^{2} K_{4} /\left(4 L^{2}\right)$ is the buckling load of a homogeneous beam with the effective bending stiffness of $K_{4}$ and length of $2 L$ [19]. Although the asymmetric bilayer composite structure bends instantly when an axial load is applied, equation (23) still predicts very large deflection at this load, which will be beyond the applicability range of the model. $K_{2}$ is thus chosen as $-0.9 \pi^{2} K_{4} /\left(4 L^{2}\right)<$ $K_{2}<0.9 \pi^{2} K_{4} /\left(4 L^{2}\right)$. As shown in the two 3D plots, the deflection at the center can be the same with different loading combinations of $K_{1}$ and $K_{2}$, so is the curvature. While, for the different loading combinations of $K_{1}$ and $K_{2}$ with the

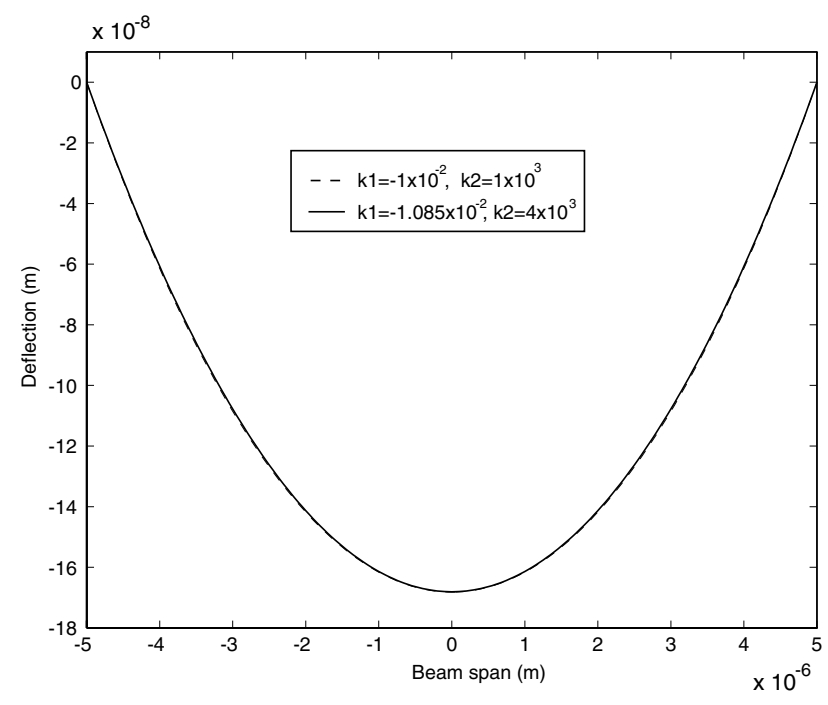

Figure 11. The deflection comparison of a hinged-hinged beam under two different loadings of $K_{1}=-1 \times 10^{-2} \mathrm{~N}, K_{2}=1 \times$ $10^{3} \mathrm{~N} \mathrm{~m}^{-1}$ and $K_{1}=-1.085 \times 10^{-2} \mathrm{~N}, K_{2}=4 \times 10^{3} \mathrm{~N} \mathrm{~m}^{-1}$, respectively. The beam length is $2 L=10 \mu \mathrm{m}$.

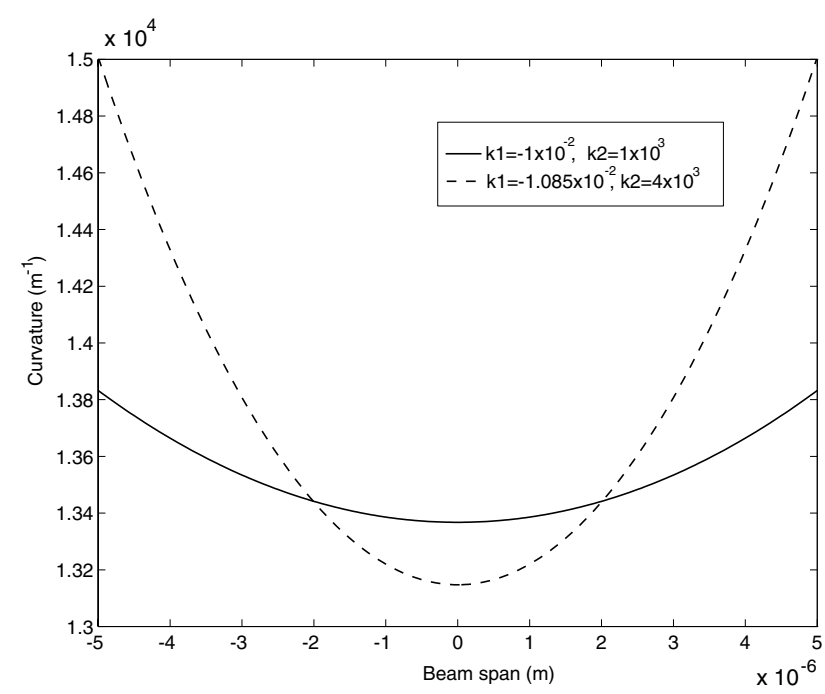

Figure 12. The curvature comparison of a hinged-hinged beam under two different loadings of $K_{1}=-1 \times 10^{-2} \mathrm{~N}, K_{2}=1 \times$ $10^{3} \mathrm{~N} \mathrm{~m}^{-1}$ and $K_{1}=-1.085 \times 10^{-2} \mathrm{~N}, K_{2}=4 \times 10^{3} \mathrm{~N} \mathrm{~m}^{-1}$, respectively. The beam length is $2 L=10 \mu \mathrm{m}$.

same deflection, their corresponding curvatures are different and vice versa. So measuring both deflection and curvature (or calculating curvature from measured deflection curve) can uniquely determine the bilayer stress state.

In the above discussions, the stress state actually means the effective bending moment $\left(K_{1}\right)$ and effective axial loading $\left(K_{2}\right)$ acting on the film-substrate bilayer. Equations (29) to (35) are the equations of relating deflection and curvatures to $K_{1}$ and $K_{2}$, not directly to $\sigma_{k}$ and $\Delta_{k} . \quad \sigma_{k}$ and $\Delta_{k}$ are related to $K_{1}$ and $K_{2}$ via equation (9). If the film thickness $\left(h_{f}\right)$ or substrate thickness $\left(h_{s}\right)$ or both are systematically varied, different sets of $K_{1}$ and $K_{2}$ will be determined from the measured deflection and curvature data of the structures with different thicknesses. For example, if there are $N$ gradient 


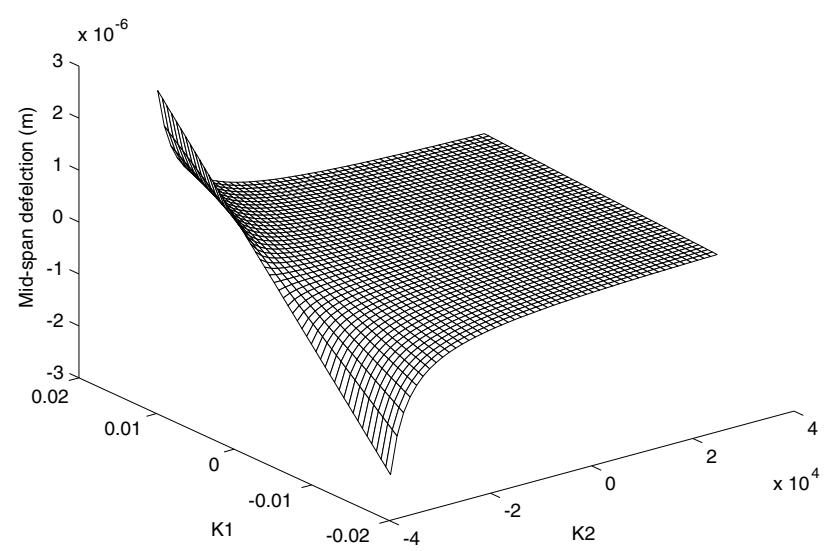

Figure 13. The variation of the displacement at the mid-span $(v(0))$ of a hinged-hinged beam as a function of $K_{1}$ and $K_{2}$.

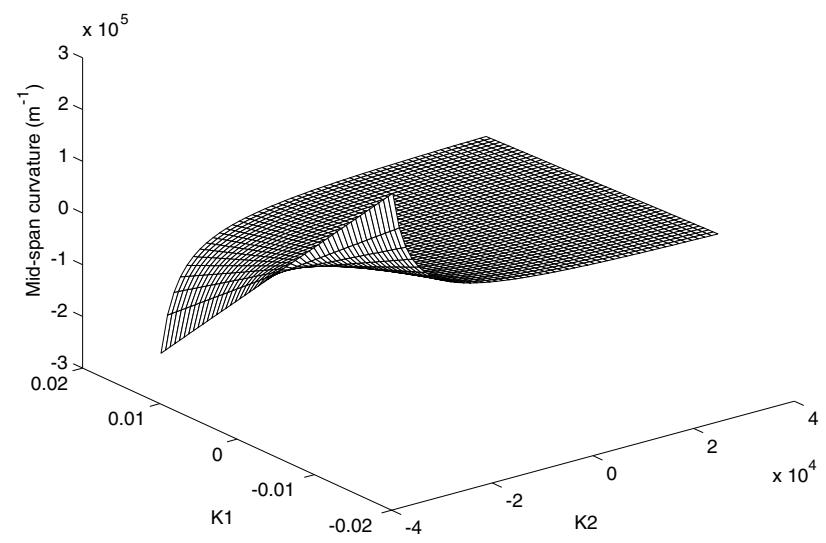

Figure 14. The variation of the curvature at the mid-span $\left(v_{x x}(0)\right)$ of a hinged-hinged beam as a function of $K_{1}$ and $K_{2}$.

stresses $\left(\sigma_{k}(k=0\right.$ to $\left.N-1)\right)$ in film and $N$ gradient stresses $\left(\Delta_{k}(k=0\right.$ to $\left.N-1)\right)$ in the substrate, total $N$ structures with $N$ different thicknesses are needed to obtain $N$ sets of different $K_{1}$ and $K_{2}$. With total $2 N$ different $K_{1}$ and $K_{2}$ obtained from $N$ different structures, it is a linear algebra problem to find $2 N$ unknowns of $\sigma_{k}$ and $\left(\Delta_{k}(k=0\right.$ to $\left.N-1)\right)$ from equation (9). This is the exact method used by Yang et al to determine the residual gradient stresses [15].

\section{Summary}

In this paper, the deflection and curvature of a film-substrate bilayer with the presence of gradient stress are derived with and without the assumption of uniform curvature. The previous derivation of assuming uniform curvature does not incorporate the effect of axial loading, which can either stiffen or soften the structure and thus influence both deflection and curvature. In the modeling analysis, whether the curvature across the whole beam is uniform or not is dependent on the kinematic assumption. With the proper kinematic assumption, the curvature is shown to be dependent on length, location, boundary conditions and axial loading. For the cantilever, hinged-hinged and free-free beams analyzed in this paper, the assumption of uniform curvature can only be valid without the presence of resultant axial loading. For the clampedhinged beam, the assumption of uniform curvature is never valid whatever the axial loading is. During the experimental measurement on the film-substrate bilayer with gradient stress, it will be useful to notice the following facts: the length and boundary conditions can influence the curvature; the curvature may vary along the span and the curvatures at different locations can be significantly different; deflection alone or curvature alone may not be sufficient to determine the residual gradient stress state.

\section{Acknowledgments}

This work is supported by both the National Natural Science Foundation of China (NSFC, grant no 10502050) and the Scientific Research Foundation for the Returned Overseas Chinese Scholars, State Education Ministry of China.

\section{References}

[1] Srikar V T and Spearing S M 2003 A critical review of microscale mechanical testing methods used in the design of microelectromechanical systems Exp. Mech. 43 238-47

[2] Fang W and Wickert J A 1995 Comments on measuring thin-film stresses using bi-layer micromachined beams J. Micromech. Microeng. 5 276-81

[3] Fang W and Wickert J A 1996 Determining mean and gradient residual stresses in thin films using micromachined cantilevers J. Micromech. Microeng. 6 301-9

[4] Elbrecht L, Storm U, Catanescu R and Binder J 1997 Comparison of stress measurement techniques in surface micromaching J. Micromech. Microeng. 7 151-4

[5] Polymenakos S, Stergiou V C, Kontos A G, Tsamis C, Raptis Y and Tsoukalas D 2002 Influence of Ge implantation on the mechanical properties of polycrystalline silicon microstructures J. Micromech. Microeng. 12 450-7

[6] Noyan I C, Murray C E, Chey J S and Goldsmith C C 2004 Finite size effect in stress analysis of interconnect structures Appl. Phys. Lett. 85 724-6

[7] Zhang X, Misra A, Wang H, Lima A L, Hundley M F and Hoagland R G 2005 Effects of deposition parameters on residual stresses, hardness and electrical resistivity of nanoscale twinned 330 stainless steel thin film J. Appl. Phys. 97094302

[8] Liau Z L 1997 Strained interface of lattice mismatch wafer fusion Phys. Rev. B 55 12899-901

[9] Freund L B and Suresh S 2003 Thin Film Materials: Stress, Defect Formation and Surface Evolution (Cambridge: Cambridge University Press)

[10] Freund L B, Floro J A and Chason E 1999 Extensions of the Stoney formula for substrate curvature to configurations with thin substrates or large deformation Appl. Phys. Lett. 74 1987-9

[11] Vanamu G, Khraishi T A and Datye A 2005 A model for curvature in film-substrate system Mater. Res. Soc. Symp. Proc. 875 97-102

[12] Vanamu G, Robbins J, Khraishi T A, Datye A and Zaidi S H 2005 Modeling and experiments on epitaxially grown multilayers with implications to critical thickness J. Electron. Mater. 34 522-7

[13] Wakeland P, Khraishi T and Zubia D 2003 Modeling and experiments on epitaxially grown multilayers with implications to critical thickness J. Electron. Mater. 32 $836-41$

[14] Zubia D, Hersee S D and Khraishi T 2002 Strain partitioning in coherent compliant heterostructures Appl. Phys. Lett. $80740-2$ 
[15] Yang E H, Yang S S and Yoo S H 1995 A technique for quantitative determination of the profile of the residual stress along the depth of $\mathrm{p}^{+}$silicon films Appl. Phys. Lett. 67 912-4

[16] Chu W-H, Mehregany M and Mullen R L 1993 Analysis of tip deflection and force of a bimetallic cantilever microactuator J. Micromech. Microeng. 3 4-7

[17] Evans D R and Craig V S J 2006 Sensing cantilever beam bending by the optical lever technique and its application to surface stress J. Phys. Chem. B 110 5450-61

[18] Huang Sh and Zhang X 2006 Extension of the Stoney formula for film-substrate system with gradient stress for MEMS applications J. Micromech. Microeng. 16 382-9

[19] Chajes A 1974 Principles of Structural Stability Theory (Englewood Cliffs, NJ: Prentice-Hall) pp 28-32

[20] Fang W and Wickert J A 1994 Post buckling of micromachined beams J. Micromech. Microeng. 4 116-22

[21] Nicu L, Temple-Boyer P, Bergaud C, Scheid E and Martines A 1999 Energy study of buckled micromachined beams for thin-film stress measurements applied to $\mathrm{SiO}_{2}$ J. Micromech. Microeng. 9 414-21

[22] Allen M G, Mehregany M, How R T and Senturia S D 1987 Microfabricated structures for the in-situ measurement of residual stress, Young's modulus, and ultimate strain of thin film Appl. Phys. Lett. 51 241-3

[23] Osterberg P M and Senturia S D 1997 M-test: a test chip for MEMS material property measurements using electrostatically actuated test structures J. Microelectromech. Syst. 6 107-17
[24] Li B, Tang X, Xie H and Zhang X 2004 Strain analysis in MEMS/NEMS structures and devices by using focused ion beam system Sensors Actuators A 111 57-62

[25] Zhang Y and Zhao Y 2006 An effective method of determining the residual stress gradients in a micro-cantilever Microsyst. Technol. 12 357-64

[26] Stoney G G 1909 The tension of metallic films deposited by electrolysis Proc. R. Soc. A 82 172-5

[27] Hu Y Y and Huang W M 2004 Elastic and elastic-plastic analysis of multilayer thin film: close-form solutions J. Appl. Phys. 96 4154-60

[28] Zhang Y, Ren Q and Zhao Y 2004 Modeling analysis of surface stress on a rectangular cantilever beam J. Phys. D: Appl. Phys. 37 2140-5

[29] Zhang Y and Zhao Y 2006 Applicability range of Stoney's formula and modified formulas for a film/substrate bilayer J. Appl. Phys. 99053513

[30] Klein C A 2000 How accurate are Stoney's equation and recent modifications $J$. Appl. Phys. 88 5487-9

[31] Timoshenko S P and Woinowsky-Krieger S 1959 Theory of Plates and Shells 2nd edn (New York: McGraw-Hill)

[32] Beer F P and Johnson E R Jr 1981 Mechanics of Material (New York: McGraw-Hill) pp 168-70

[33] Timoshenko S P 1925 Analysis of bi-metal thermostats J. Opt. Soc. Am. 11 233-55

[34] Thurman A L and Mote C D 1969 Free, periodic, nonlinear oscillation of an axially moving strip J. Appl. Mech. 36 83-9 